\title{
CONCEPT OF OPERATIONS TO SYSTEM DESIGN AND DEVELOPMENT-AN INTEGRATED SYSTEM FOR AIRCRAFT MISSION FEASIBILITY ANALYSIS USING STK ENGINE, MATLAB AND LABVIEW
}

\author{
Princy Randhawa ${ }^{1}$ and Vijay Shanthagiri ${ }^{2}$ \\ Assistant Professor, Department of Mechatronics Engineering, Manipal University Jaipur \\ Principle System Engineer, Oeuvre Software Solutions, Bangalore
}

\begin{abstract}
In recent times, there has been a significant rise in usage of aircrafts in surveillance and reconnaissance missions. Not all the aircrafts survive the harsh testing conditions put forth by the enemy regions. Aircraft Survivability Analysis gives the measure of the chances of survival for different counter strategies. The mission would be recalculated if particular sortie does not fall within the physical boundary of the performance of an aircraft. This is required both for the success of the mission and the survivability of the aircraft in the harsh enemy conditions.

A system is envisioned comprising of the accurate modeling of the physical world and the accurate model of control system. An interoperable system which can work seamlessly together will provide mission planners, System integrators, aeronautical/aerospace engineers a milieu wherein the Control System designer who is found wanted as far as the physical world is concerned is given a system which can simulate the real world in lab conditions. To achieve this, we combine the two most promising environments prevalent in the industry today namely Systems tool kit for modeling the operational environment MATLAB and LabVIEW for modeling the control system environment. Using a Math script window of LabVIEW, we have designed the aircraft model and controlling the variables of an aircraft using a simulation loop of a LabVIEW. The different flight conditions were arrived using Orthogonal Array $(O A)$ based on different Aircraft weight, Altitude, Mach number configurations. This attempts to span the aircrafts across the regimes in aircrafts flight envelope. A system comprising of both, with seamless UDP based connection between the two is developed to expedite the process of development of feasible control system design and verification which allows the aircrafts to undertake complex mission. This system we believe would answer questions of limits of the aircrafts maneuverability and survivability in terms of its limitation concerning control system design and development of commercial fighter aircrafts, UAV's and Quad copters.
\end{abstract}

INDEX TERMS

Aerospace control, Aircraft navigation,

\section{KEYWORDS}

Aircrafts, Control System, Navigation guidance and control, Aircraft mission planning Line-of-sight, Systems Tool Kit, LabVIEW, Control design, Closed Loop System.

DOI : $10.5121 /$ ijics.2015.5401 


\section{INTRODUCTION}

To connect the physical world with the control system environment is to have given the designer a debugging feature unlike never before. It is advantageous if an attempt to finalize a control system design is supported or criticized not by heuristics or intuitive insights based on experience but by a real system which models a system connected to a control system design environment. While all these days, the feedback loop would ensure stability of a process or a system, it is assured here that a feedback for verifying a systems stability, controllability, observability is verified for different dynamically created mission paths and not just a small set of paths which are usually available due to lack of simulating environment. In other words, this paper is about connecting a highly accurate simulating environment such as STK with a highly accurate and user friendly Instrument Design and Control system design environment such as LabVIEW.

\section{Mission Planning On Systems Tool Kit}

Systems Tool Kit the user with the following important inputs for simulating the mission path

* Scenario configuration: - Set Units; Set Epoch; Set Time Period

* Way Point Creation: - Click and Create Waypoints on Aircraft mission modeler; plan CAP mission, Refueling, Reconnaissance mission and other critical mission paths

* Animation setup and control: - Animate Set-Values, Set-Time; Start Forward

* Vehicle attitude initialization example:-Set-Attitude */Launch Vehicle/L1 Profile Fixed Euler 91.1 80.9 0.0 323 "Facility/WFF_Pad_1 Body".

* Aircraft simulated live data: - Get position of each simulated point and other values pertaining to aerodynamic attributes such as Pitch, Yaw, and Roll Turn Radius in time.

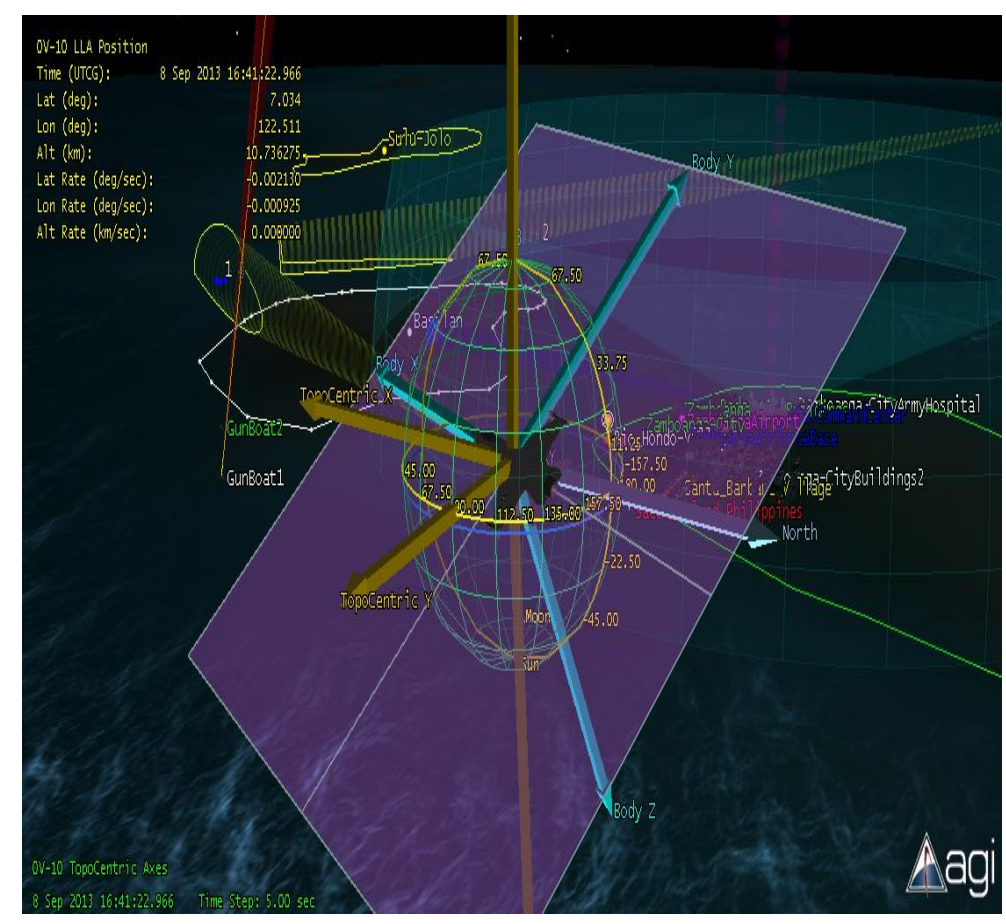

Fig 1: STK's Simulation Environment concerning Aircrafts Attitude, Pitch, Roll and Yaw. 
International Journal of Instrumentation and Control Systems (IJICS) Vol.5, No.4, October 2015

\section{Aircraft Control System Design On Labview}

* LabVIEW is a graphical programming environment that has very powerful numeric array and string handling capabilities, and easy numeric to string conversion.

* LabVIEW has good capability to model complex control system design. It provides user friendly virtual instrumentation environment to iterate and dynamically update control values into it.

* Building user interface is very simple.

* Basic TCP functions built in - Open, Write, Read, Close Connection.

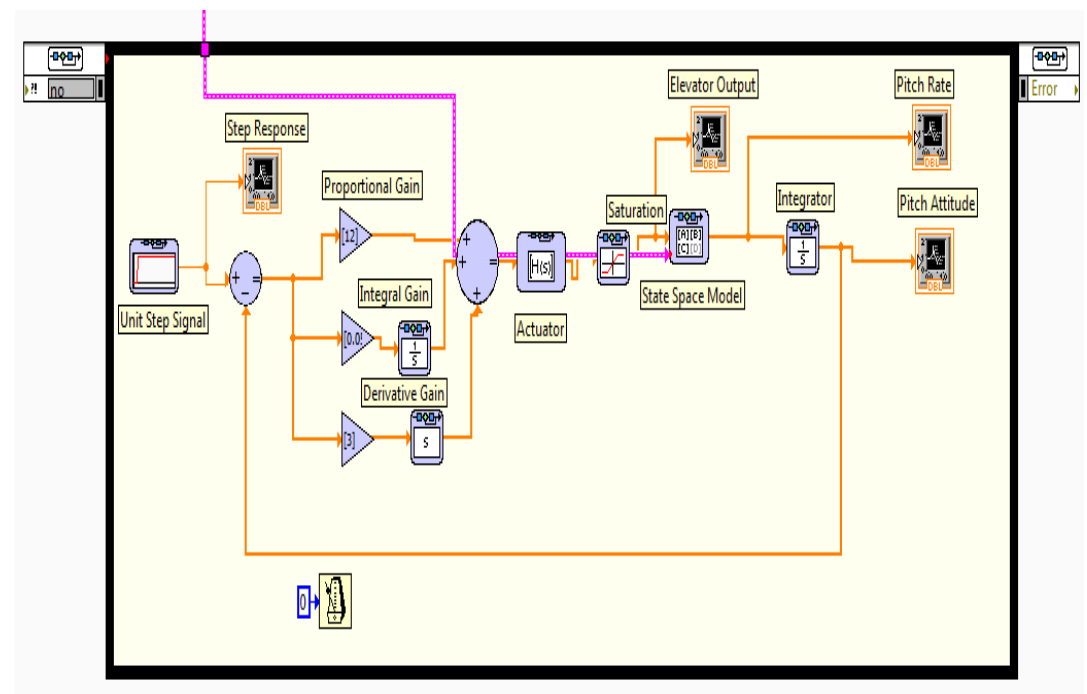

Fig 2: LabVIEW Design and Simulation Environment with Control System Block Diagram

\section{INTEGRATING STK AND LABVIEW}

* It is envisioned that STK will connected to LabVIEW using UDP Connection. Since the data rate is not too high TCP/IP connection can also be used.

* The events leading to invocation and information exchange between STK and LabVIEW will be delineated.

* The Message structures with coded heading will be agreed between both the parties.

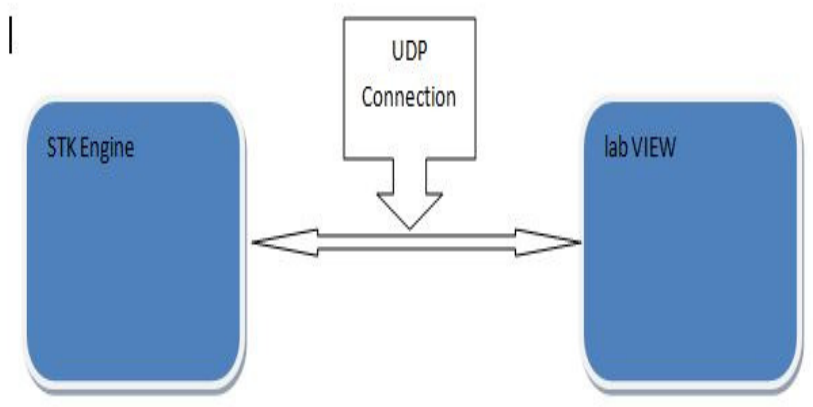

Fig. 3: Block Diagram of the integrated system and the information flow. 
International Journal of Instrumentation and Control Systems (IJICS) Vol.5, No.4, October 2015

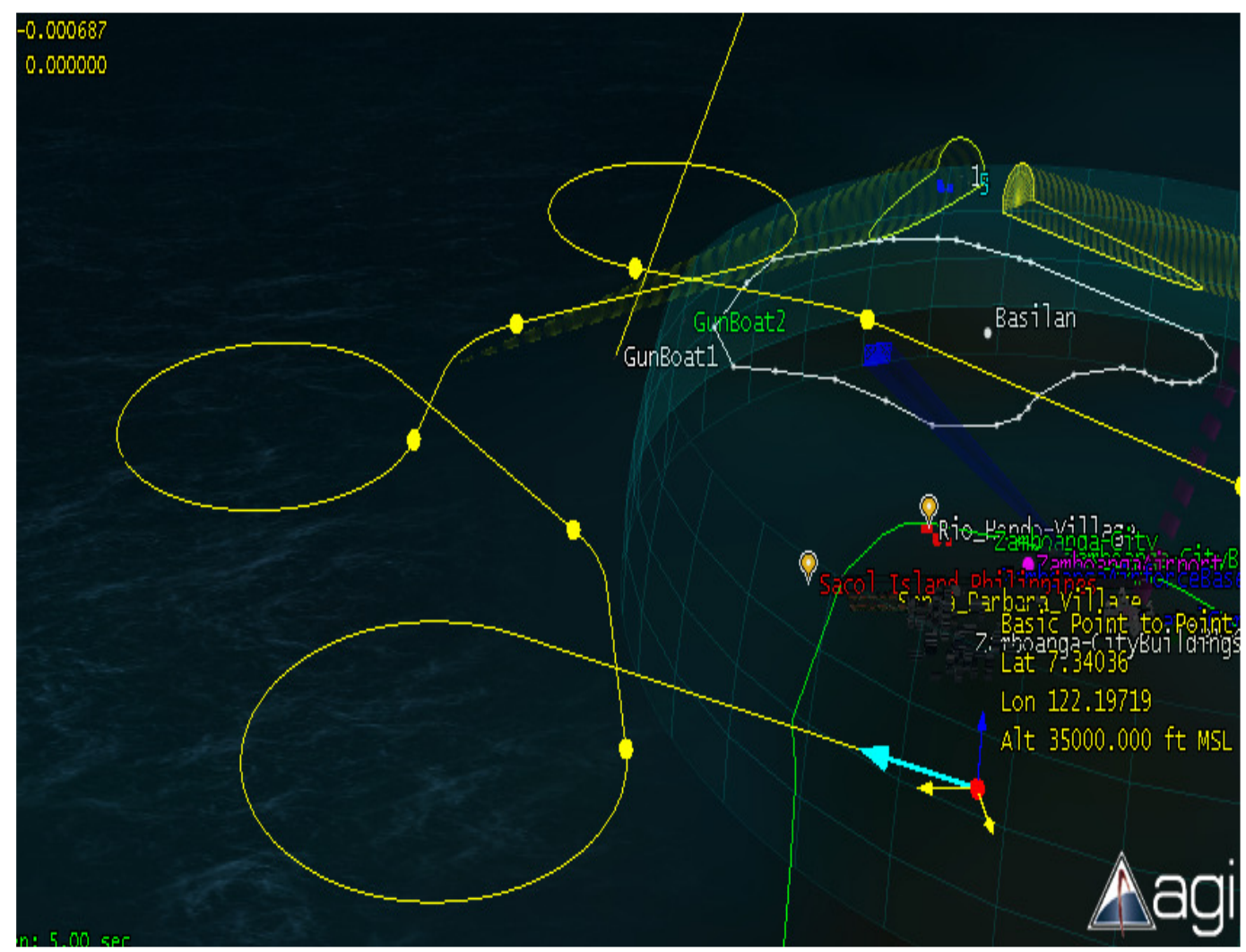

Fig. 4: STK's Simulation Environment concerning Waypoint Creation of Aircrafts

The mutual data flow will be though structures containing the information. A Message Structure of TYPE1 is as follows.

\section{[Message Struct Type 1]}

$\left\{\begin{array}{l}\quad \text { int16 Message Header } \\ \text { int16 Message Type } \\ \text { char[50] AicraftName } \\ \text { double Latitude } \\ \text { double Longitude } \\ \text { double Pitch } \\ \text { double Roll } \\ \text { double Yaw } \\ \text { double Pitch Rate } \\ \text { double Roll Rate } \\ \text { double Yaw Rate } \\ \text { double Velocity } \\ \text { double Epoch Time }\end{array}\right.$

The message is received by LabVIEW over a preselected socket and interpreted. 
International Journal of Instrumentation and Control Systems (IJICS) Vol.5, No.4, October 2015

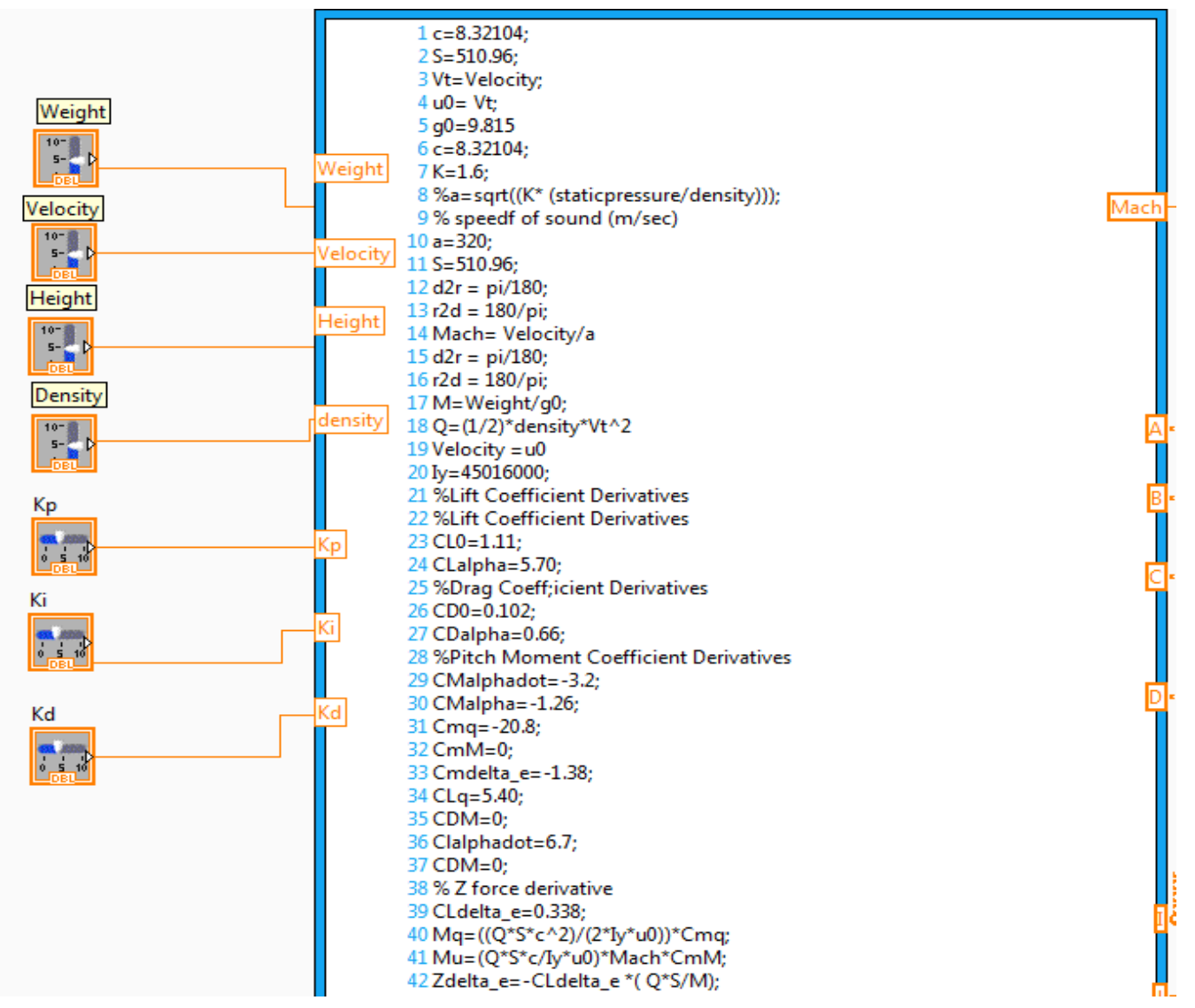

Fig. 5: Behind the scenes of LabVIEW where the Control System Schema is rigged to receive external inputs through instruments assigned to produce the signals.

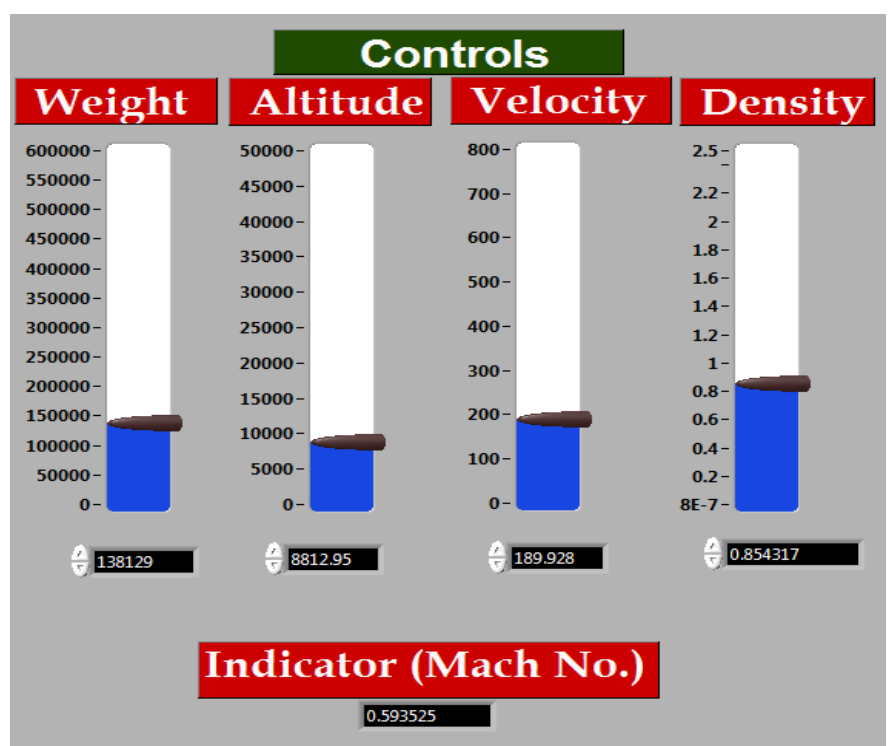

Fig. 6: Dynamic Data Creation Panel where data is received though a socket and connected 
International Journal of Instrumentation and Control Systems (IJICS) Vol.5, No.4, October 2015

The information flow between STK and LabVIEW is shown below.STK used here with its engine and a c\# based application is used to invoke the engine. This makes it easy to gain full control over the data transfer without too much UI interaction for the user. The way points created as shown in Fig 6 is directly converted and sent over the socket to Lab View's port for stability analysis of the path.

The sequential flow between STK engine and LabVIEW is shown below.

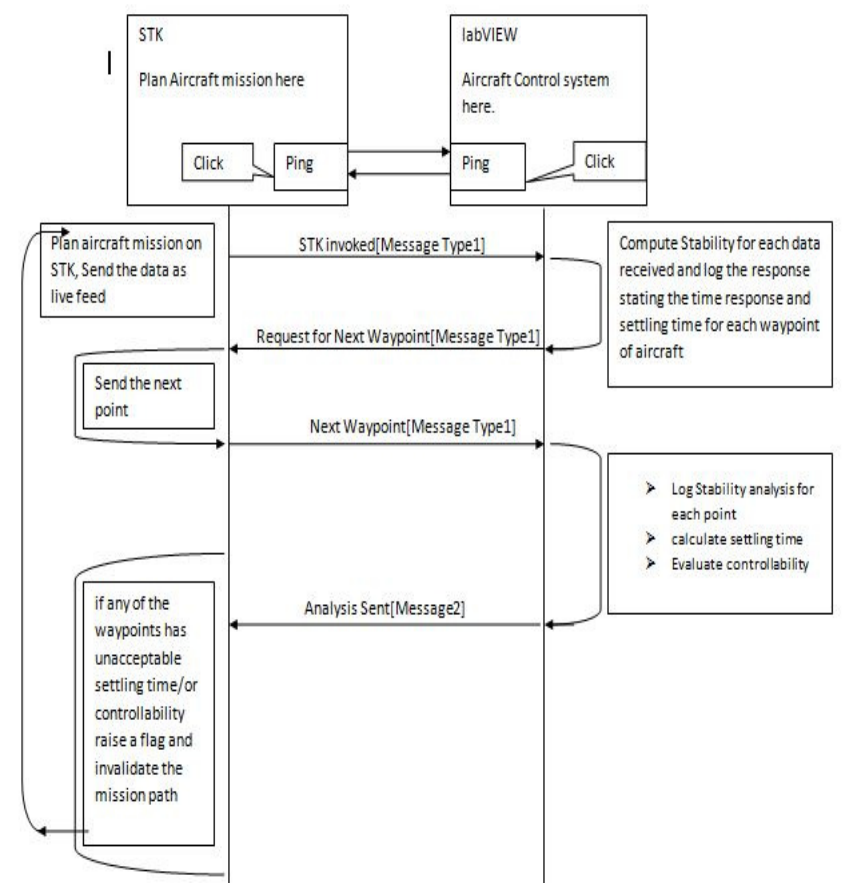

Fig. 7: Sequence Diagram of the integrated system and the information flow

\section{Use Case With Results - Control System Design Of A Commercial Aircraft USIng StK AND LabVieW}

For designing a plant model through LabVIEW Simulation loop, we need a longitudinal equation. For the dynamics of a longitudinal aircraft, we need variables which are (small) deviations from operating point or trim conditions. These small deviations are created by first modelling the aircraft path on Systems Took Kit and then streaming the delta change in $\mathrm{u}, \alpha, \theta$,q with respect to time.

- $\quad \mathrm{u}$ : velocity of aircraft along body axis

- $\alpha$ : angle of attack (the angle between the velocity vector and the $x$-axis of the aircraft

- $\theta$ : Angle between body axis and horizontal (up is positive)

$\mathrm{q}=\dot{\theta}:$ Angular velocity of aircraft (pitch rate) 
International Journal of Instrumentation and Control Systems (IJICS) Vol.5, No.4, October 2015

\section{INPUTS}

Control or actuator inputs:

- $\quad \delta e$ : Elevator angle ( $\delta e>0$ is down)

If we introduce the longitudinal state variable vector

$$
\mathbf{x}=\left[\begin{array}{lll}
\mathbf{u} & \boldsymbol{q} & \mathbf{q}
\end{array}\right]
$$

and the longitudinal control vector

$$
u(t)=\left[\begin{array}{ll}
\delta e & 0
\end{array}\right.
$$

These equations are equivalent to the system of first-order equations $\quad \operatorname{In} \dot{x}(t)=A x(t)+B u(t)$

$$
y(t)=C(t)+D u(t)
$$

where: $A-$ State matrix $(\mathrm{n} \times n)$

$B$-Input matrix $(\mathrm{n} \times m)$

$C$-Output matrix $(\mathrm{r} \times n)$

$D$-Direct matrix $(\mathrm{r} \times m)$

$x(t)$ - column vector of $\mathrm{n}$ state variables

$u(t)$ - column vector of $m$ input variables

$y(t)$ - column vector of $\mathrm{n}$ output variables

$x$ represent the time derivative of the state vector $\mathrm{x}$, and the matrices appearing in this equation are

$\mathbf{A n}=\left[\begin{array}{cccc}X u & X \alpha & 0 & -g_{0} \cos \theta_{0} \theta \\ \frac{Z_{u}}{u_{0}} & \frac{Z_{\alpha}}{u_{0}} & \frac{Z_{0}+u_{0}}{u_{0}} & -\frac{g_{0} \sin \theta_{0} \theta}{u_{0}} \\ M_{u} & M_{\alpha} & M_{q} & 0 \\ 0 & 0 & 1 & 0\end{array}\right]$

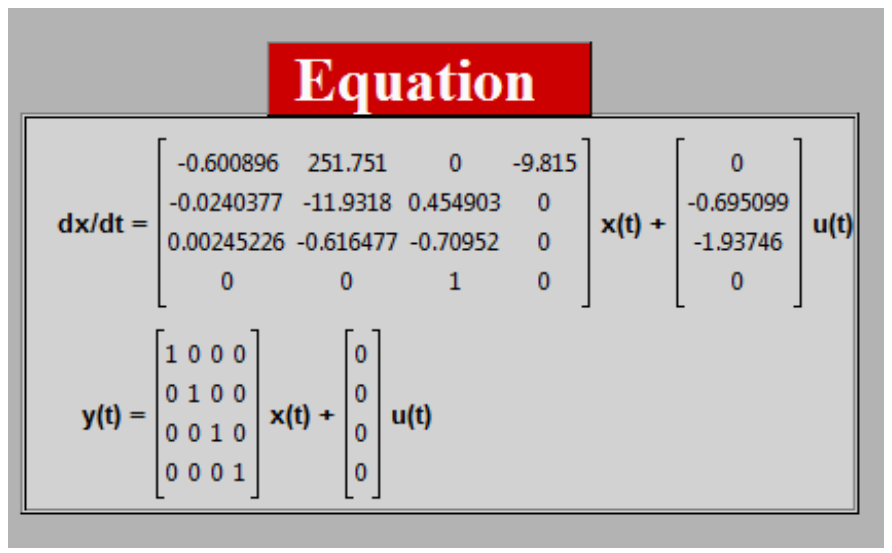

Fig. 8: State Space Equation of an Aircraft plant model 
International Journal of Instrumentation and Control Systems (IJICS) Vol.5, No.4, October 2015

Thus, transfer function for output variable $\mathrm{u}$ with respect to elevator deflection is presented in Fig $9 \frac{u(s)}{\delta e(s)}$

$$
\frac{1.721 e-015 s^{\wedge} 3-0.2504 s^{\wedge} 2-0.5849 s+0.01012}{s^{\wedge} 4+0.2388 s^{\wedge} 3+0.8295 s^{\wedge} 2+0.005087 s+0.0008952}
$$

Thus, transfer function for output variable $\alpha$ with respect to elevator is presented as follows shown in Fig $9 \frac{\alpha(s)}{\delta e(s)} \quad \frac{-0.003388 s^{\wedge} 3-0.009529 s^{\wedge} 2 \_0.001072 s-0.0009804}{s^{\wedge} 4+0.2388 s^{\wedge} 3+0.8295 s^{\wedge} 2+0.005087 s+0.0008952}$

Thus, transfer function for output variable $\mathrm{q}$ with respect to elevator deflection is presented as shown in Fig 10. $\frac{q(s)}{\delta e(s)} \quad \frac{-0.009151 s^{\wedge} 3-0.002106 s^{\wedge} 2-0.007502 s+8.674 e-019}{s^{\wedge} 4+0.2388 s^{\wedge} 3+0.8295 s^{\wedge} 2+0.005087 s+0.0008952}$

Thus, transfer function for output variable $\theta$ with respect to elevator deflection is presented and shown in Fig $10 \frac{\theta(s)}{\delta e(s)} \frac{-5.551 e-017 s^{\wedge} 3-0.009151 s^{\wedge} 2-0.002106 s-0.007502}{s^{\wedge} 4+0.2388 s^{\wedge} 3+0.8295 s^{\wedge} 2+0.001754 s+3.613 e-006}$

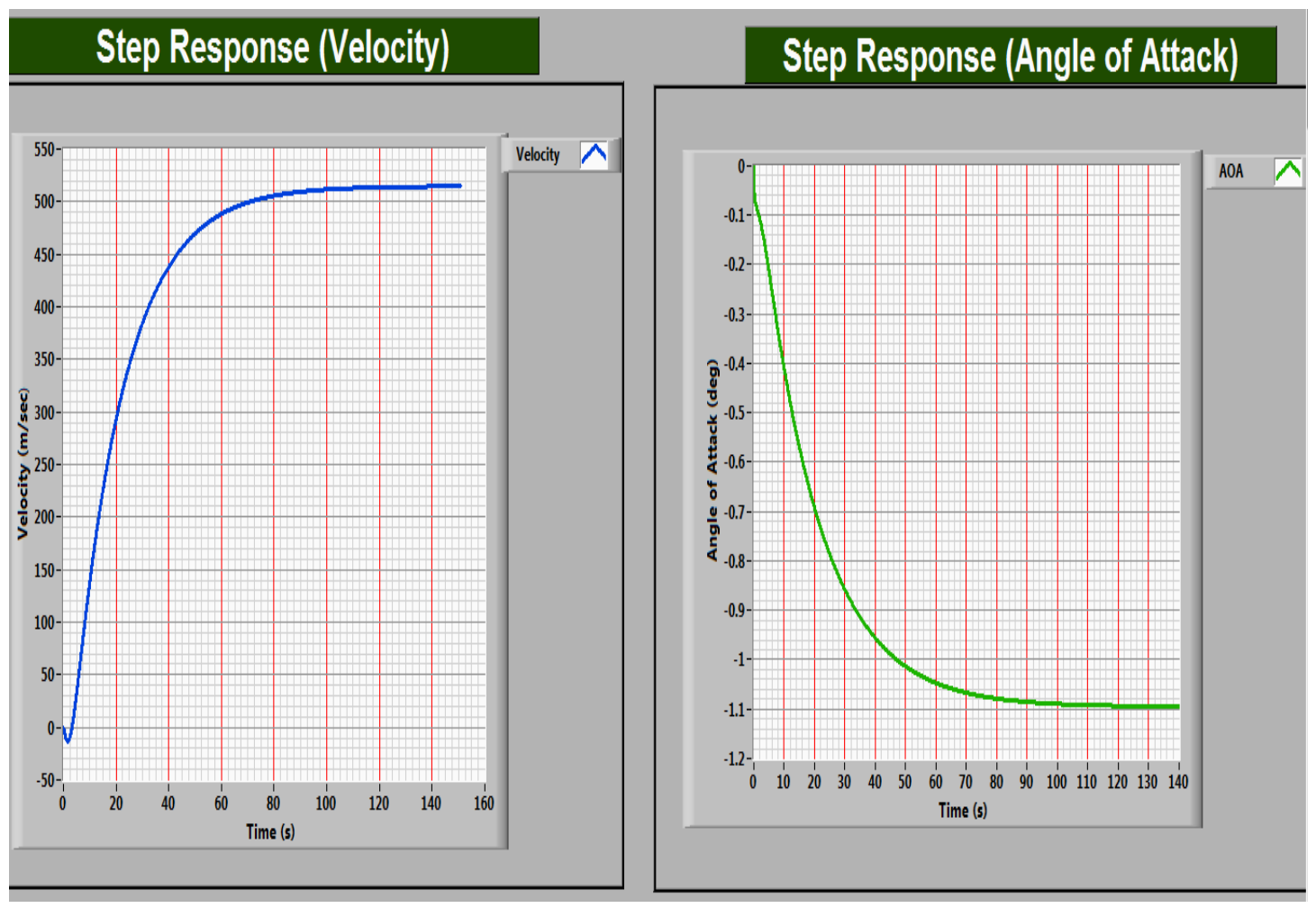

Fig 9: Longitudinal Aircraft Response for velocity (m/se) and angle of attack (deg) v/s time (sec) to 1 deg Elevator Step Input 


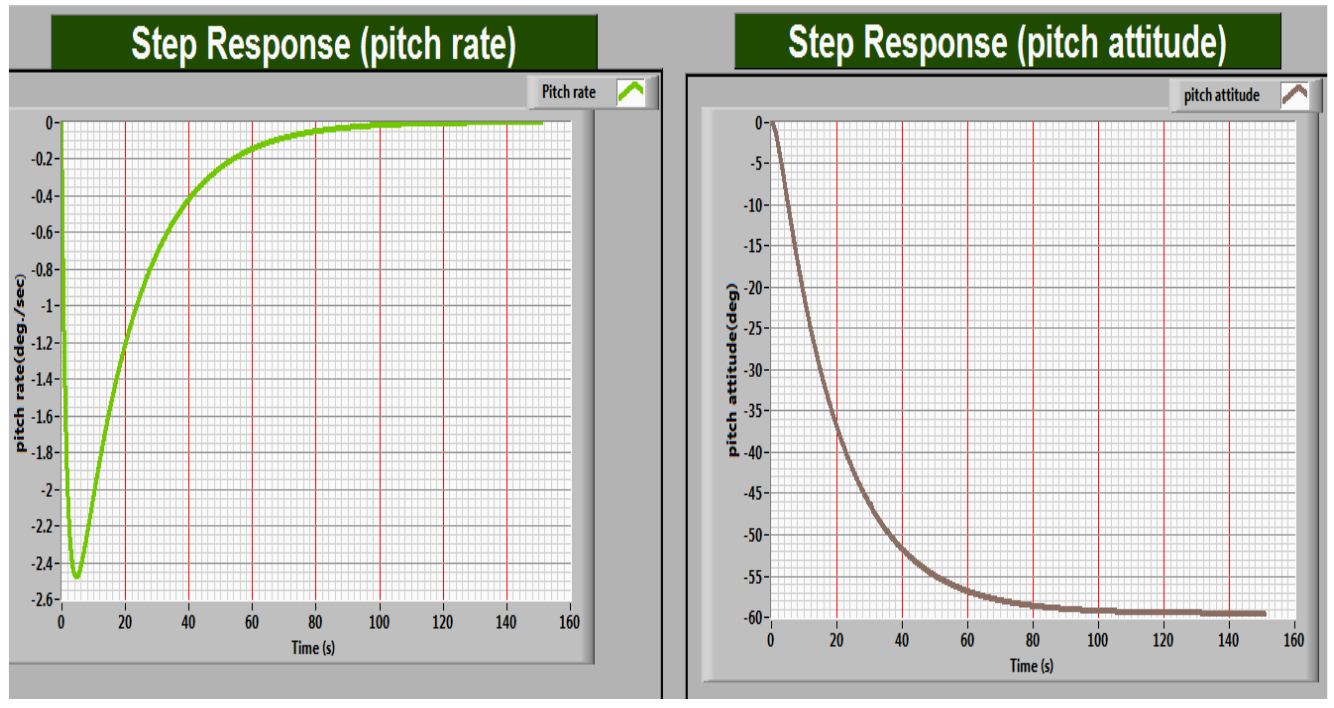

Fig 10: Longitudinal Aircraft Response for pitch rate (deg/se) and pitch attitude (deg) v/s time (sec) to 1 deg Elevator Step Input

We will begin with an analysis of how an aircraft will behave in the pitch when it is controlled solely by the elevator. Figure 11 shows the poles are on the L.H.S determines the stability of plant. The poles near to the origin show Phugoid mode and the poles far from the origin shows short period mode. It infers that at very high weight with less altitude, the Mach no. should be less (velocity). It was assumed that the control surfaces (elevator) have certain limits on the deflection. Figure 12 shows the typical variations of elevator deflection versus aircraft speed to maintain aircraft longitudinal trim. Figure 13 shows the response of elevator control defection of 1-deg step input for

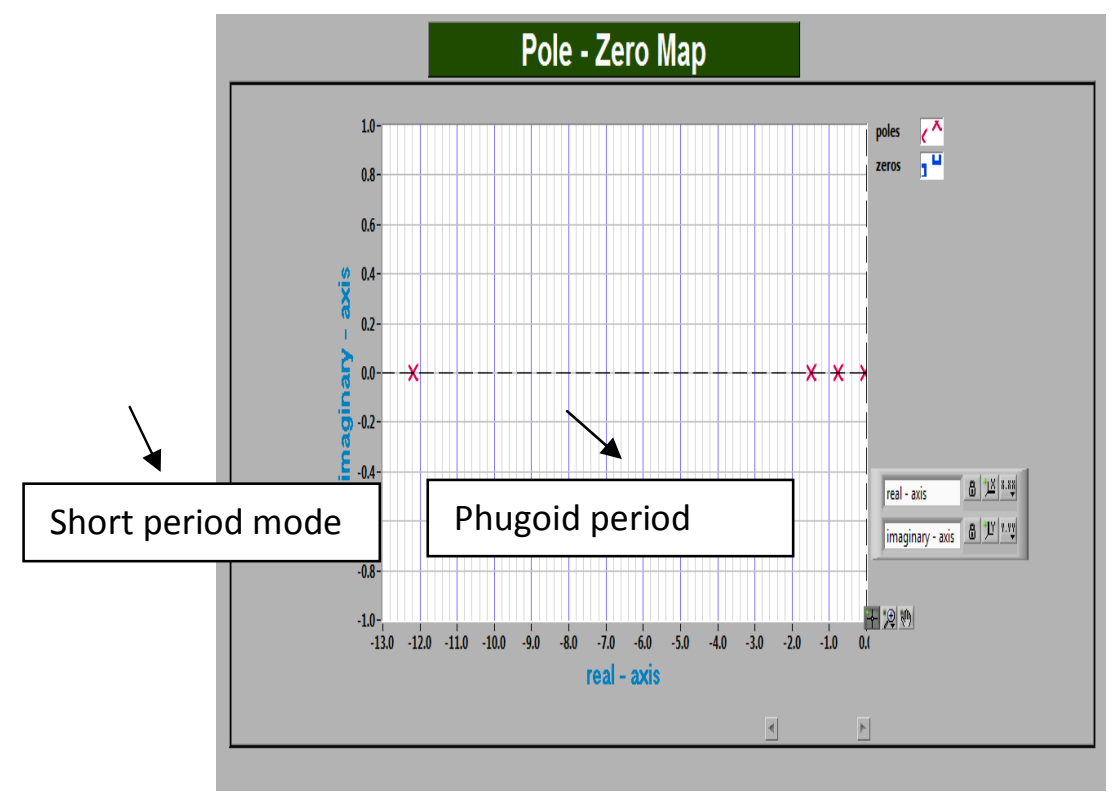

Fig. 11: Pole Zero map of short period and Phugoid mode. 
International Journal of Instrumentation and Control Systems (IJICS) Vol.5, No.4, October 2015

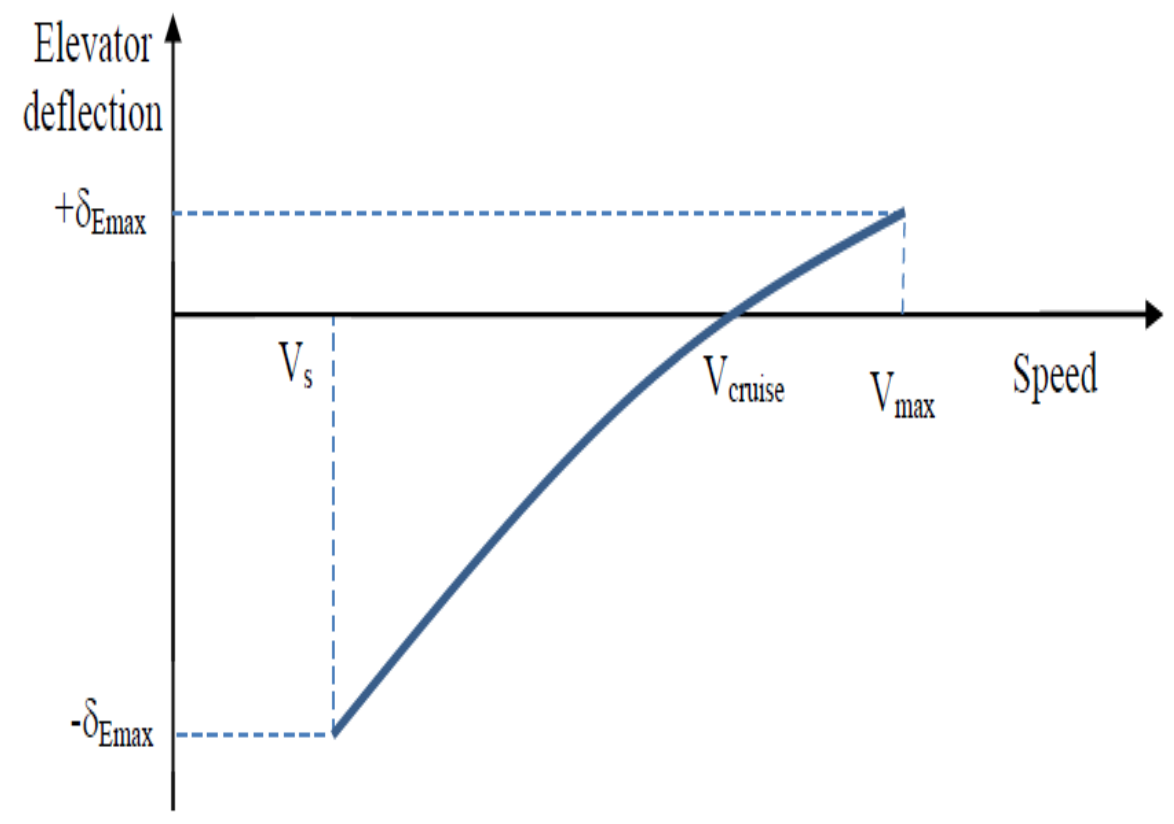

Fig. 12: Typical variations of elevator deflection versus aircraft speed

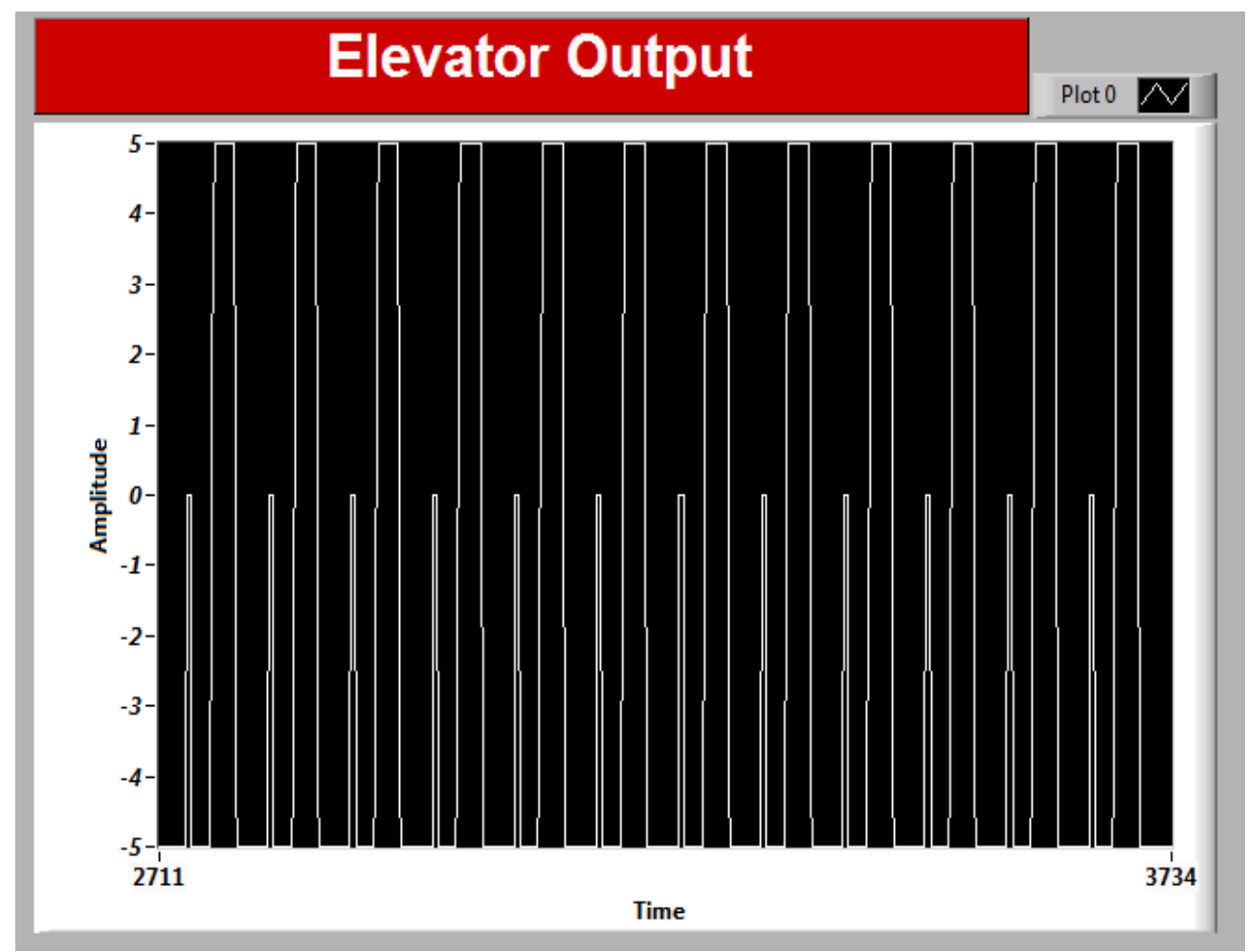

Fig. 13: Elevator control deflection for 1-deg Elevator step Input 


\section{Is System Controllable of Not?}

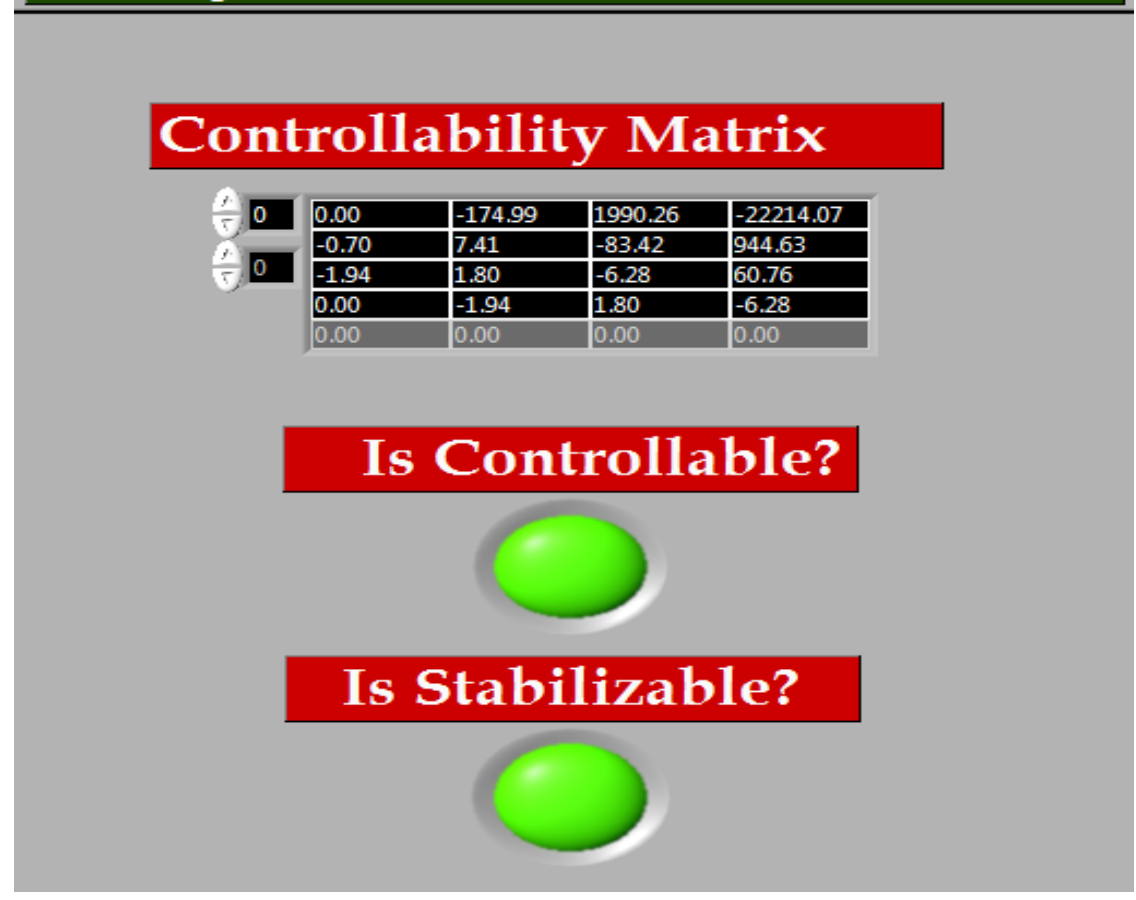

Fig. 14: Controllability Matrix

Figure 14 shows the controllability matrix which depends on the coefficients matrixes A and B which states that plant can be controllable by seeing control input. if $\mathrm{Qc} \neq 0$ (Controllable) or vice versa.

It must be noted here that this above calculation is repeated for every input from STK to LabVIEW. The math script which models the system is invoked repeatedly for newer values and a feed back is sent as to whether the performance of the system is within the expected ranges of stability and controllability. A system which can respond successfully to almost all kinds of mission paths can then be given a go for production of prototype and field testing.

It is easy to note here that the effort to integrate STK, LabVIEW along with Math script ensures that the mission/operations of various kinds are tested before the agency or organization invests in real hardware development.

\section{Applicability}

The application of such a system spans a whole lot of applications. It is particularly valid in following use cases

1. Design of Quad Copters with mounted Direction Finder.

2. Design of Drones with validated Survivability analysis in enemy environment.

3. Design of UAV for Intelligence Surveillance and Reconnaissance missions where maneuvering is remotely done on a UAV which is far from the friendly territory 
International Journal of Instrumentation and Control Systems (IJICS) Vol.5, No.4, October 2015

\section{FUTURE WORK}

With this framework in place, industries can extract the best of STK and LabVIEW. In future, the same type of set up can be used in the following areas.

1. Satellite Constellation Design

2. Satellite Swarm Design

3. Aircraft Control and Air Defense analysis and validation

4. Model validation of proposed design against uncertainties in the natural environments

\section{CONCLuSion}

With the above results it can be inferred that system development can be expedited by connecting a simulation environment with Development environment to get a feed back about one's own design. The quick results during mission planning ensure that the design which has been approved for final construction or even a prototype construction is indeed closer to the desired design. This helps largely in reducing development time and saves energy and repetition of work. More importantly, it is well know that system design , structural design of aircrafts and flying object involves large sum of money in construction. What might work in lab condition using LabVIEW might not work in the real condition in nature due to uncertain condition and different convoluted use cases which might show up in the real world scenario. here we have brought the real world scenario to the laboratory so that through use cases are created to validate the design against different mission plans, aircraft paths to test the response of the control systems against the path it has to endure.

\section{REFERENCES}

[1] Harsh Kaji, Shruti Annigeri,*Prafulla Patil. "Designing PID Controller using LabVIEW for Controlling Fluid Level of Vessel", International Journal of Engineering Research and Applications (IJERA) ISSN: 2248-9622 Vol. 3, Issue 2, March -April 2013, pp.1329-1333.

[2] Ricardo Dunia (NI), Eric Dean (NI), and Dr. Thomas Edgar (UT),Introduction to LabVIEW for Control Design \& Simulation", Process Dynamics and Control 2nd edition, by Seborg, Edgar, Mellichamp, Wiley 2004.

[3] Nilar Lwin, Hla Myo Tun, “ Implementation Of Flight Control System Based On Kalman And PID Controller For UAV” International journal of scientific \& technology research volume 3, issue 4, April 2014 ISSN 2277-8616.

[4] Marc. R., 2001, “A SIMULINK Toolbox for Flight Dynamics and Control Analysis”. FDC 1.2, 2nd Edition..

[5] Ihnseok. R., Sangook. C., Sanghyuk. P., Keeyoung. C., 2012, “Autopilot Design for a Target Drone using Rate Gyros and GPS”, International Journal of Aeronautical \& Space Sci., Vol. 13, No. 4.

[6] Fouad Yacef1, Hana Boudjedir1, Hicham Khebbache2, and Omar Bouhali1, "From PID to State Feedback Attitude Stabilization of a Quadrotor UAV", International Journal of Information Technology, Control and Automation (IJITCA) Vol.2, No.3, July 2012.

[7] Jan Roskan, "Airplane flight dynamics and automatic flight control”, DAR Corporation, 1998 USA.

[8 ] Lance sherry, Michael Feary, Randall Mumaw, "A cognitive engineering analysis of the vertical navigation (VNAV) function”, NASA report, 12 January 2000 